\title{
Misclassification of exposure is high when interview data on drug use are used as a proxy measure of chronic drug use during follow-up
}

\author{
Annette B. Beiderbeck ${ }^{\mathrm{a}, \mathrm{b}}$, Miriam C.J.M. Sturkenboom ${ }^{\mathrm{b}}$, Jan W.W. Coebergh ${ }^{\mathrm{b}}$, \\ Hubert G.M. Leufkens ${ }^{\mathrm{a}}$, Bruno H.Ch. Stricker ${ }^{\mathrm{b}, *}$ \\ ${ }^{a}$ Department of Pharmacoepidemiology \& Pharmacotherapy, Utrecht Institute for Pharmaceutical Sciences (UIPS), Utrecht, The Netherlands \\ ${ }^{\mathrm{b}}$ Pharmaco-epidemiology Unit, Departments of Epidemiology \& Biostatistics and Internal Medicine, Erasmus University Medical Center, \\ PO Box 1738, 3000 DR Rotterdam, The Netherlands
}

Accepted 8 December 2003

\begin{abstract}
Background and objective: In many observational studies, the association between drugs and disease is analyzed with information from a baseline interview. We investigated the magnitude and direction of exposure misclassification by comparing interview data at baseline with prospectively gathered pharmacy data.

Methods: The study population for this study consisted of a cohort of 2,487 participants aged 71 years or older from the Rotterdam Study. Data on drug use were gathered at the baseline interview and through pharmacies during the follow-up period between January 1, 1991, and January 1, 1999. We assessed the sensitivity, specificity, and positive and negative predictive value of interview data as proxy measures of chronic use of calcium channel blockers (CCB) in comparison with longitudinal medication records from the pharmacy.

Results: Only 3 of the 206 subjects (1.5\%) who reported use at baseline did not use CCBs during follow-up. Of the 2,281 persons who reported no use of CCBs at baseline, however, 354 actually used CCBs during follow-up (15.5\%). The difference between interview data and pharmacy records corresponded to a misclassification bias of 0.73 (95\%CI: $0.52-1.02$ ).

Conclusion: Misclassification of exposure was high when interview data were used as a proxy measure of chronic use during follow-up. (C) 2004 Elsevier Inc. All rights reserved.
\end{abstract}

Keywords: Calcium channel blockers; Cancer; Exposure assessment; Misclassification; Validity; Pharmacoepidemiology

\section{Introduction}

Earlier cohort studies on the association between calcium channel blockers (CCBs) and cancer used cross-sectional assessment of exposure at the start of follow-up, with contradictory results [1-4]. In the first prospective cohort study to suggest that CCBs may cause cancer, drug exposure was assessed at baseline [3]. Obviously, it is highly unlikely that exposure to $\mathrm{CCBs}$ on one single day alone plays a role in the induction of cancer. Hence, the implicit assumption is that exposure to CCBs during interview is a proxy of chronic use during follow-up. Unless drug exposure is defined as a time-dependent covariate, this assumption is the direct consequence of statistical modeling with a traditional Cox proportional hazards model in which a baseline characteristic is assumed to remain present during follow-up $[5,6]$. Under everyday circumstances, however, it is quite common that

* Corresponding author. Tel.: +31-10-408-7489; fax: +31-10-408-9382.

E-mail address: b.stricker@erasmusmc.nl (B.H.Ch. Stricker). subjects who were nonexposed at baseline start treatment during follow-up. Similarly, others may discontinue the drug of interest or change its intake during follow-up. Hence, the exposure status will almost always change over time. An adequate epidemiological study requires data on drug exposure that are specific regarding type, formulation, and timing of exposure [7,8]. Misclassification of exposure will mostly lead to biased effect estimates while the magnitude and direction of this bias depend on the pattern of misclassification [9]. Most authors discard the problem of exposure misclassification as relatively unimportant as long as it is nondifferential (i.e., independent of disease status). The argument is that this leads to conservative risk estimates [10], a fact which is generally considered as preferable to inflated estimates. The objective of any epidemiological study is a valid risk estimation, so such an argument is not completely satisfactory, because both underestimation and overestimation of the true risk are undesirable.

Our objective was to explore the degree of misclassification of exposure to CCBs during follow-up by crosssectional assessment at baseline interview. We therefore 
conducted a study to quantify the magnitude and direction of misclassification introduced by baseline assessment of CCB exposure in an earlier study that prospectively investigated the association between CCBs and cancer [4].

\section{Methods}

\subsection{Study population}

We used data from the Rotterdam Study, a prospective, population-based cohort study of neurological, cardiovascular, locomotor, and ophthalmologic diseases in the elderly [11]. In brief, all inhabitants of Ommoord aged 55 years or older who were living in this suburb of Rotterdam were invited in 1990-1993 to participate in the study. Of the 10,275 eligible subjects, 7,983 (78\%) participated and were interviewed at home. During this visit, trained interviewers administered an extensive questionnaire covering, among other topics, socioeconomic background, food intake, medical history, and current medication use at the day of interview.

All study subjects obtain their prescribed medicines through one of the seven pharmacies serving the Ommoord region and surroundings; the pharmacies are fully automated and share one computer network. Over a 5-yearperiod, approximately $98 \%$ of the study population obtained at least one prescription through one of these pharmacies. The pharmacy records contain automated information on all dispensed prescriptions since January 1, 1991, and are virtually complete for all prescribed drugs in this population.

The population for the present study consisted of the cohort members who were included in our earlier study on the association between CCBs and cancer [4]. The potential study period consisted of an 8-year period between January 1, 1991, and January 1, 1999. We excluded all patients with a history of cancer before 1991. To account for an induction plus latent period of at least 1 year, we also excluded all patients who had a tumor that was diagnosed in the first year of follow-up (January 1, 1991-January 1, 1992) and all patients who had filled a prescription for a cytostatic agent before January 1, 1992. We also excluded all participants below 71 years of age at study entry, all patients who were not registered with one of the pharmacies and all persons who were interviewed before we had pharmacy data. The remaining population was followed from January 1, 1992, until one of the following events: a registration of malignant cancer (ICD-9-CM codes 140 through 208), death, transfer to another area, or end of the study period, whichever of the events occurred earliest.

\subsection{Drug exposure assessment}

We compared data on use of CCBs during the baseline interview with longitudinal pharmacy data during follow-up.

\subsubsection{Interview data}

Exposure to CCBs was assessed during the baseline interview. At that time, patients were asked to show containers for all drugs, both prescription and over-the-counter, taken at any time during the 2 weeks preceding the interview.

\subsubsection{Pharmacy data}

The pharmacy records contained automated information on all dispensed prescriptions since January 1, 1991; these include the product name, the international nonproprietary name, the Anatomical Therapeutic Chemical (ATC) code, the quantity dispensed (i.e., number of filled tablets/capsules or other dosage form), the date of dispensing, the prescribed daily number, the dosage, and the legend duration (prescription length). From the pharmacy records, we identified all prescriptions for CCBs. We distinguished three duration categories: nonuse (no prescription), nonchronic use $(<180$ days), and chronic use ( $\geqslant 180$ days).

\subsection{Analysis}

Our objective was to quantify the magnitude and direction of exposure misclassification as a consequence of crosssectional assessment of exposure by interview at baseline. Our specific interest was to see whether baseline assessment of exposure to CCBs would be a good proxy for actual cumulative exposure to CCBs during follow-up, as this is the implicit assumption in a traditional Cox proportional hazards model. To quantify the degree of misclassification of CCB exposure we calculated the sensitivity, specificity, and positive and negative predictive values (PPV, NPV) for interview by reference to pharmacy data, which were considered as a gold standard [12]. These measures were calculated for nonchronic and chronic use of CCBs during follow-up.

For comparisons of proportions, we used $\chi^{2}$ statistics or a Fisher exact test whenever the expected cell count was less than 5. Because age and gender are strong risk factors for cancer, we examined differences among the sensitivity, specificity, PPV, and NPV within strata of these variables. The direction and extent of misclassification was measured by comparing the hazard ratio from a time-varying Cox proportional hazards model of chronic exposure to CCBs and cancer based on either interview or pharmacy data. We defined time-varying cumulative exposure variables on the basis of pharmacy records from the date of baseline interview or January 1, 1992, until the end of follow-up as described earlier [4]. Misclassification of exposure was assessed and expressed in a quality index in which the ratio of the biased risk estimate and its gold standard plus $95 \%$ confidence limits are calculated [13].

\section{Results}

The final study cohort consisted of 2,487 persons, with a median of 7.6 years of follow-up.

The characteristics of the study population at baseline are listed in Table 1. The 206 participants taking CCBs 
Table 1

Characteristics of the study population at baseline $(N=2,487)$

\begin{tabular}{|c|c|c|c|c|c|}
\hline \multirow[b]{2}{*}{ Characteristic } & \multicolumn{2}{|c|}{ Users of CCB $(n=206)$} & \multicolumn{2}{|c|}{ Nonusers of CCB $(n=2,281)$} & \multirow[b]{2}{*}{$P$-value } \\
\hline & $N$ & $\%$ & $N$ & $\%$ & \\
\hline \multicolumn{6}{|l|}{ Age } \\
\hline$\leqslant 75$ & 70 & 34 & 807 & 35 & \\
\hline $76-80$ & 57 & 28 & 605 & 27 & \\
\hline $81-85$ & 41 & 20 & 424 & 19 & \\
\hline$>85$ & 38 & 18 & 445 & 20 & .9 \\
\hline Women & 131 & 64 & 1,527 & 68 & .2 \\
\hline Cancer & 24 & 12 & 204 & 9 & .2 \\
\hline Hypertension & 44 & 21 & 547 & 24 & 6 \\
\hline Ischemic heart disease & 63 & 31 & 294 & 13 & .001 \\
\hline Coronary bypass ( $1-5$ events) & 9 & 4 & 48 & 2 & .02 \\
\hline PTCA (1-5 events) & 4 & 2 & 11 & 1 & .004 \\
\hline History of stroke & 18 & 9 & 125 & 6 & .06 \\
\hline Angina pectoris & 42 & 20 & 159 & 7 & .001 \\
\hline Peripheral artery disease & 59 & 29 & 503 & 22 & .07 \\
\hline Diabetes mellitus & 40 & 19 & 308 & 14 & .06 \\
\hline Heart failure & 18 & 9 & 113 & 5 & .05 \\
\hline \multicolumn{6}{|l|}{ Calcium channel blockers } \\
\hline Verapamil & 50 & 24 & - & & \\
\hline Diltiazem & 77 & 37 & - & & \\
\hline Nifedipine & 64 & 31 & - & & \\
\hline \multicolumn{6}{|l|}{ Co-medication } \\
\hline Diuretics & 75 & 36 & 518 & 23 & .001 \\
\hline$\beta$-blocking agents & 49 & 24 & 323 & 14 & .001 \\
\hline ACE inhibitors & 28 & 14 & 142 & 6 & .001 \\
\hline Statins & 7 & 3 & 19 & 1 & .002 \\
\hline Systemic corticosteroids & 10 & 5 & 58 & 3 & .1 \\
\hline
\end{tabular}

Abbreviations: ACE, angiotensin converting enzyme; CCB, calcium channel blocker; PTCA, percutaneous transluminal coronary angioplasty.

were significantly more likely to suffer from diabetes mellitus, cardiovascular diseases, and a history of stroke than the 2,281 nonusers. They also more frequently used other cardiovascular drugs and statins.

At baseline, $206(8.3 \%)$ persons reported use of CCBs during the past 2 weeks. Only 3 of the 206 subjects $(1.5 \%)$ who reported CCB use at baseline did not use CCBs during follow-up (PPV $=98.5 \%$ ) (Table 2 ). The number of persons with a false-negative exposure status, however, was relatively high compared with the number of persons with a falsepositive exposure status during follow-up. The sensitivity of interview data were below $50 \%$ for any exposure, as well

Table 2

Validity of interview-based exposure measurement for the assessment of exposure to CCBs

\begin{tabular}{|c|c|c|c|c|}
\hline \multirow[b]{3}{*}{ User status, interview } & \multicolumn{4}{|c|}{ User status, pharmacy } \\
\hline & \multicolumn{2}{|l|}{ Users } & \multirow[b]{2}{*}{ Nonusers } & \multirow[b]{2}{*}{ Total } \\
\hline & Chronic & Nonchronic & & \\
\hline User & 195 & 8 & 3 & 206 \\
\hline Nonuser & 204 & 150 & 1,927 & 2,281 \\
\hline Total & 399 & 158 & 1,930 & 2,487 \\
\hline
\end{tabular}

Validity measures for any use: Positive predictive value (PPV), $98.5 \%$; negative predictive value (NPV), $84.5 \%$; sensitivity, $36.4 \%$; specificity, $99.8 \%$.

Validity measures for chronic use: PPV, 94.7\%; NPV, 89.4\%; sensitivity, $48.8 \%$; specificity, $99.5 \%$. as for chronic exposure (Table 2). Of the 2,281 persons who reported no use of CCBs at baseline, 354 actually used CCBs during follow-up (15.5\%) and 204 (57.6\%) of them were chronic users ( $\geqslant 180$ days of cumulative use during followup). Hence, the negative predictive value was $84.5 \%$ for any CCB use during follow-up and $89.4 \%$ for chronic use.

Table 3 shows the validity parameters of interview data as a proxy for chronic exposure to $\mathrm{CCBs}$ by age, gender and cancer status. The positive predictive value did not differ significantly among different age groups, but the sensitivity and negative predictive value of the interview data varied highly with age. The negative predictive value of the baseline interview increased with age from $89.1 \%$ among those $<75$ years of age to $96.2 \%$ in persons $>85$ years (Table 3 ). The sensitivity changed from $43.5 \%$ to $68.5 \%(P=.009)$. The validity of interview data did not differ by gender. The sensitivity of interview data as proxy for chronic exposure to CCBs during follow-up differed between persons with cancer and those without cancer (60\% and $47.8 \%$, respectively), but did not reach statistical significance $(P=.17)$.

To estimate the effect of exposure misclassification, we assessed the association between calcium channel blocker use and cancer with the two different exposure assessment methods. Based on cross-sectional assessment of exposure to $\mathrm{CCBs}$ at baseline the age and gender adjusted relative risk was 1.4 for use of CCBs (95\% CI 0.9-2.1). In contrast, the age and gender adjusted relative risks based on nonchronic 
Table 3

Validity of interview-based exposure measurement for the assessment of chronic exposure to CCB, stratified by age, gender, and cancer

\begin{tabular}{|c|c|c|c|c|c|c|c|c|}
\hline & \multirow[b]{2}{*}{ User status, interview } & \multirow[b]{2}{*}{ Chronic user } & \multirow[b]{2}{*}{ Nonuser $^{a}$} & \multirow[b]{2}{*}{ Total } & \multicolumn{4}{|c|}{ Validity measures for interview data as a proxy measure for chronic exposure } \\
\hline & & & & & PPV & NPV & Sensitivity & Specificity \\
\hline \multicolumn{9}{|l|}{ Age } \\
\hline \multirow[t]{2}{*}{$\leqslant 75 \mathrm{yr}$} & User & 68 & 2 & 70 & $97.1 \%$ & $89.1 \%$ & $43.6 \%$ & $99.7 \%$ \\
\hline & Nonuser & 88 & 719 & 807 & & & & \\
\hline \multirow[t]{2}{*}{$76-80 \mathrm{yr}$} & User & 52 & 5 & 57 & $91.2 \%$ & $89.8 \%$ & $45.6 \%$ & $99.1 \%$ \\
\hline & Nonuser & 62 & 543 & 605 & & & & \\
\hline \multirow[t]{2}{*}{$81-85 \mathrm{yr}$} & User & 38 & 3 & 41 & $92.7 \%$ & $91.3 \%$ & $50.7 \%$ & $99.2 \%$ \\
\hline & Nonuser & 37 & 387 & 424 & & & & \\
\hline \multirow[t]{2}{*}{$>85 \mathrm{yr}$} & User & 37 & 1 & 38 & $97.4 \%$ & $96.2 \%$ & $68.5 \%$ & $99.8 \%$ \\
\hline & Nonuser & 17 & 428 & 445 & & & & \\
\hline \multicolumn{9}{|l|}{ Sex } \\
\hline \multirow[t]{2}{*}{ Men } & User & 71 & 4 & 75 & $94.7 \%$ & $90.9 \%$ & $51.4 \%$ & $99.4 \%$ \\
\hline & Nonuser & 67 & 667 & 734 & & & & \\
\hline \multirow[t]{2}{*}{ Women } & User & 124 & 7 & 131 & $94.7 \%$ & $91.1 \%$ & $47.5 \%$ & $99.5 \%$ \\
\hline & Nonuser & 137 & 1,410 & 1,547 & & & & \\
\hline \multicolumn{9}{|l|}{ Cancer } \\
\hline \multirow[t]{2}{*}{ Yes } & User & 21 & 3 & 24 & $87.5 \%$ & $93.1 \%$ & $60.0 \%$ & $98.4 \%$ \\
\hline & Nonuser & 14 & 190 & 204 & & & & \\
\hline \multirow[t]{3}{*}{ No } & User & 174 & 8 & 182 & $95.6 \%$ & $90.9 \%$ & $47.8 \%$ & $99.6 \%$ \\
\hline & Nonuser & 190 & 1,887 & 2,077 & & & & \\
\hline & Total & 399 & 2,088 & 2,487 & & & & \\
\hline
\end{tabular}

Abbreviations: NPV, negative predictive value; PPV, positive predictive value.

${ }^{a}$ Never user or user for less than 180 cumulative days of exposure.

and chronic exposure according to pharmacy data were 1.1 (95\% CI 0.68-1.9) and 0.9 (95\% CI 0.60-1.2), respectively. The crude relative risks for cancer according to interview data and pharmacy records were 1.30 and 0.95 , respectively. This corresponds to a quality index of $0.95 / 1.30$, which corresponds to a misclassification bias of $0.73(95 \% \mathrm{CI}$ 0.52-1.02).

\section{Discussion}

We found that cross-sectional assessment of calcium channel blocker exposure by means of a baseline interview was not a good proxy measure of actual use during follow-up. The extent of exposure misclassification was substantial, especially because of the high proportion of false-negatives in persons who were nonuser at baseline but started drug use during follow-up. This had a negative influence on the sensitivities and negative predictive values. Because the sensitivities of interview data as a proxy for any use or chronic use differed among persons who later developed cancer, misclassification was differential. As a consequence, the relative risk of CCB use was overestimated when based on cross-sectional interview data and changed from 1.4 to a relative risk of 1.1 for nonchronic use, and 0.9 for chronic use, when longitudinal data were used. Although this decrease of $36 \%$ was not statistically significant, such a difference may be substantial in larger studies. It endorses the theoretical argument that assessment of exposure on a continuous basis is probably a better proxy of the actual exposure status than assessment on one particular day.
In epidemiological studies on the association between drug use and cancer, drug use is often assessed cross-sectionally during a baseline interview [2,3,14,15]. Although misclassification may be less substantial in studies in which a change in exposure status by reference to repeated interviews is taken into account $[14,16]$, it is obvious that the use of continuous data on drug intake is preferable. Such data from pharmacy records or reimbursement data facilitate the use of time-dependent variables in the analysis. In a traditional Cox proportional hazards model, baseline determinants are used on the assumption that such variables are constant over time [5]. This assumption is valid for determinants such as blood group and gender, but not for drugs. In our study, baseline interview data led to relatively large numbers of false-negatively classified subjects for chronic use of CCBs. It is unlikely that incidental use of a calcium channel blocker causes cancer, so only chronic use can be associated with cancer in a meaningful way.

This study aimed to assess misclassification by comparing interview data with pharmacy records. Pharmacy records can be regarded as a virtually complete source of drug exposure and have been used previously to assess completeness of medical records or interview data $[17,18]$. Although the fact that a patient fills a prescription at the pharmacy does not guarantee that the drug is taken, adherence to chronic therapy is easily checked by timely refills. A patient who regularly refills his drug is usually compliant. A limitation of our study might be the arbitrary definition of chronic drug use as more than 180 days of cumulative use. It is obvious, however, that with other definitions of chronic use sensitivity will also be low, because a baseline interview can never tell which nonusers will start the drug in the future. 
The validity estimates obtained in this study might be helpful in the design or interpretation of other studies on calcium channel blocker use and risk of cancer. Even if misclassification in these studies was independent of disease status, risk estimates will be biased towards a more conservative estimate [19]. We found, however, that age (an important risk factor for cancer) and case status were associated with the validity of interview data. This resulted in an overestimation of the risk for CCBs when exposure was assessed by interview. Indeed, the hazard ratio of cancer associated with use of CCBs based on cross-sectional assessment was higher than when assessed by pharmacy data. This suggests that the conflicting results of studies on the association between calcium channel blocker use and cancer might partly be explained by misclassification bias.

In conclusion, cross-sectional exposure ascertainment at baseline as a measure for chronic exposure might result in biased risk estimates of disease. Unlike confounding, misclassification cannot be controlled for in the analysis $[10,20]$. Preferably, continuous data on drug intake should be used if chronic exposure is the major parameter of interest and if the follow-up period is long.

\section{References}

[1] Trenkwalder P, Hendricks P, Hense HW. Treatment with calcium antagonists does not increase the risk of fatal or non-fatal cancer in an elderly mid-European population: results from STEPHY II. Starnberg Study on Epidemiology of Parkinsonism and Hypertension in the Elderly. J Hypertens 1998;16:1113-6.

[2] Pahor M, Guralnik JM, Salive ME, Corti MC, Carbonin P, Havlik RJ. Do calcium channel blockers increase the risk of cancer? Am J Hypertens 1996;9:695-9.

[3] Pahor M, Guralnik JM, Ferrucci L, Corti MC, Salive ME, Cerhan JR, Wallace RB, Havlik RJ. Calcium-channel blockade and incidence of cancer in aged populations. Lancet 1996;348:493-7.

[4] Beiderbeck-Noll AB, Sturkenboom MCJM, van der Linden PD, Herings RMC, Hofman A, Coebergh JWW, Leufkens HGM, Stricker $\mathrm{BHCh}$. Verapamil is associated with an increased risk of cancer in the elderly: the Rotterdam Study. Eur J Cancer 2003;39:98-105.
[5] Cox DR. Regression models and life tables (with discussion). J R Stat Soc Ser B 1972;34:187-220.

[6] Clayton D, Hills M. Time-varying explanatory variables. In: Statistical models in epidemiology. Oxford (UK): Oxford University Press; 1993: 307-18.

[7] Armstrong BG. Effect of measurement error on epidemiological studies of environmental and occupational exposures. Occup Environ Med 1998;55:651-6.

[8] Armstrong BK, White E, Saracci R. Principles of exposure measurement in epidemiology. Oxford (UK): Oxford University Press; 1992.

[9] van Staa TP, Abenhaim L, Leufkens H. A study of the effects of exposure misclassification due to the time-window design in pharmacoepidemiologic studies. J Clin Epidemiol 1994;47:183-9.

[10] Greenland S, Robins JM. Confounding and misclassification. Am J Epidemiol 1985;122:495-506.

[11] Hofman A, Grobbee DE, de Jong PT, van den Ouweland FA. Determinants of disease and disability in the elderly: the Rotterdam Elderly Study. Eur J Epidemiol 1991;7:403-22.

[12] Lau HS, de Boer A, Beuning KS, Porsius A. Validation of pharmacy records in drug exposure assessment. J Clin Epidemiol 1997;50: 619-25.

[13] Marshall RJ. Assessment of exposure misclassification bias in casecontrol studies using validation data. J Clin Epidemiol 1997;50:15-9.

[14] Cohen HJ, Pieper CF, Hanlon JT, Wall WE, Burchett BM, Havlik RJ. Calcium channel blockers and cancer. Am J Med 2000;108:210-5.

[15] Hole DJ, Gillis CR, McCallum IR, McInnes GT, MacKinnon PL, Meredith PA, Murray LS, Robertson JW, Lever AF. Cancer risk of hypertensive patients taking calcium antagonists. J Hypertens 1998; 16:119-24.

[16] Michels KB, Rosner BA, Walker AM, Stampfer MJ, Manson JE, Colditz GA, Hennekens CH, Willett WC. Calcium channel blockers, cancer incidence, and cancer mortality in a cohort of U.S. women: The Nurses' Health Study. Cancer 1998;83:2003-7.

[17] Sjahid SI, van der Linden PD, Stricker BH. Agreement between the pharmacy medication history and patient interview for cardiovascular drugs: the Rotterdam elderly study. Br J Clin Pharmacol 1998;45: 591-5.

[18] Heerdink ER, Leufkens HG, Koppedraaijer C, Bakker A. Information on drug use in the elderly: a comparison of pharmacy, general-practitioner and patient data. Pharm World Sci 1995;17:20-4.

[19] Flegal KM, Brownie C, Haas JD. The effects of exposure misclassification on estimates of relative risk. Am J Epidemiol 1986;123:736-51.

[20] Kosinski AS, Flanders WD. Evaluating the exposure and disease relationship with adjustment for different types of exposure misclassification: a regression approach. Stat Med 1999;18:2795-808. 